\title{
A dissociation between object manipulation spatial ability and spatial orientation ability
}

\author{
MARIA KOZHEVNIKOV and MARY HEGARTY \\ University of California, Santa Barbara, California
}

\begin{abstract}
We developed psychometric tests of spatial orientation ability, in which people are shown a twodimensional array of objects, imagine taking a perspective within the array, and indicate the direction to a target object from this perspective. Patterns of errors on these tests were consistent with experimental studies of perspective taking. Characteristic errors and verbal protocols supported the validity of the perspective-taking tests, suggesting that people encoded the objects in the display with respect to a body-centered coordinate system when the imagined perspective was more than $90^{\circ}$ different from the orientation of the display. By comparing alternative models in a confirmatory factor analysis, we found that the ability to mentally rotate and manipulate an imagined object (as measured by tests of spatial visualization and spatial relations) and the ability to reorient the imagined self (as measured by the perspective-taking tests) are separable spatial abilities.
\end{abstract}

Experimental studies of spatial reasoning provide strong evidence for a distinction between mental rotation and perspective-taking processes (e.g., Huttenlocher \& Presson, 1973, 1979; Presson, 1982; Wraga, Creem, \& Profitt, 2000). These studies compare tasks that require a person to anticipate the appearance of an array of objects after it is rotated (rotation tasks) with tasks in which a person must anticipate the appearance of a fixed array after a change in his/her perspective (orientation or perspective tasks). Although these tasks are logically equivalent, both children and adults show different patterns of errors for the two tasks. Perspective tasks lead to egocentric errors, in which the participant describes the array from his/her current perspective, rather than the imagined perspective, whereas errors in mental rotation problems are not systematic. A distinction between orientation and rotation processes has also been found in object recognition tasks, so that people have difficulty recognizing changes in object arrays after the array rotates, but not after they physically change their perspective with respect to the array (Simons \& Wang, 1998).

These results are consistent with behavioral and neuroscience evidence (D. Bryant \& Tversky, 1999; Easton \& Sholl, 1995; Muller, Kubie, Bostock, Taube, \& Quirk, 1991; O’Keefe \& Nadel, 1978; Paillard, 1991; Rieser, 1989;Zacks, Rypma, Gabrieli, Tversky, \& Glover, 1999) for a dissociation between knowledge of the spatial relations among a set of stable reference objects (object-to-

This research was supported by the Office of Naval Research under contract N00014-96-10525 to the second author. We thank Pam Freitas for help with conducting the study and Nancy Collins for statistical advice. Correspondence concerning this article should be addressed to M. Kozhevnikov, Rutgers University, Department of Psychology, 101 Warren St., Smith Hall, Newark, NJ 07102 (e-mail: maria@psychology.rutgers.edu). object representational system) and knowledge of objects' locations relative to the observer's body (self-toobject representational system). Mental rotation of an object or an array of objects involves imagining movement relative to an object-based frame of reference, which specifies the location of one object (or its parts) with respect to other objects. In contrast, imagining a different orientation (perspective) involves movement of the egocentric frame of reference, which encodes object locations with respect to the front/back, left/right, and up/down axes of the observer's body (D. Bryant \& Tversky, 1999; Easton \& Sholl, 1995; Wraga et al., 2000). Zacks et al. (1999) have shown that egocentric perspective transformations lead to increased cortical activity around the left parietal-temporal-occipital junction, whereas object-based spatial transformations, such as mental rotation, lead to an activation in posterior areas that is greater in the right hemisphere than in the left hemisphere.

In this paper, we examine whether a person's ability to mentally manipulate objects from a stationary point of view (involving an object-to object representational system) and his/her ability to imagine taking a different perspective in space (involving a self-to-object representational system) are separable mental abilities. Although the distinction between mental rotation and perspectivetaking processes has been made in experimental and neuroscience studies, it does not necessarily imply a distinction from an individual-differences perspective. That is, object manipulation and perspective taking could be separate processes but tap the same underlying ability, in the sense that when an individual is good at object manipulation, he/she is also good at spatial orientation, and vice versa.

The literature on individual differences up to now has questioned the separability of spatial abilities that involve 
imagining a change in one's own orientation in space from spatial abilities that involve mentally manipulating an object from a fixed perspective. Psychometric studies of spatial ability (e.g., Carroll, 1993; Eliot \& Smith, 1983; Lohman, 1988; McGee, 1979) report evidence for several major spatial abilities factors. Two of these factors, spatial visualization and spatial relations, require the ability to mentally manipulate spatial forms from a fixed perspective and involve the object-to-object representational system. Spatial visualization tasks require imagination of a complex sequence of mental manipulations. For example, the Paper Folding Test (shown in the Appendix) and Form Board Tests are typically loaded on the spatial visualization factor. Spatial relations tasks (also called speeded rotation) involve simpler mental manipulations, such as a single-step mental rotation of a two-dimensional object, and emphasize speed of processing (Carroll, 1993). For example, the Card Rotation Test (shown in the Appendix) typically loads on the spatial relations factor. For the purposes of the present study, we refer to tests of spatial visualization and spatial relations collectively as object manipulation spatial tests. ${ }^{1}$

A third proposed spatial factor, spatial orientation, is defined as the ability to imagine how a stimulus array will appear from another perspective. For example, the Guilford-Zimmerman Spatial Orientation Test (Guilford \& Zimmerman, 1948), the most commonly used test of spatial orientation, requires a subject to identify the position of a boat that would give a particular view of the landscape (see an example of the task in the Appendix). In contrast to spatial visualization and spatial relations tests, spatial orientation tests are designed to engage the self-to-object representational system. Some researchers (e.g., McGee, 1979) have argued that there is support for the existence of the spatial orientation factor. However, a major meta-analysis of the factor-analytic research (Carroll, 1993) failed to find evidence for the separability of this factor from spatial visualization (see also Borich \& Bauman, 1972; Price \& Eliot, 1975).

Spatial orientation might be difficult to separate from other spatial factors because tests designed to measure this ability are often solved by mentally rotating the stimulus rather than by reorienting oneself (Barratt, 1953; Carpenter \& Just, 1986; Carroll, 1993). For example, in a verbal protocol study, Barratt found that the majority of participants reported a mental rotation strategy on the Guilford-Zimmerman task. This would account for its typically high loading on the spatial visualization factor (Carroll, 1993). We suggest that the failure to find a distinction between object manipulation and spatial orientation abilities in the psychometric literature occurs because there are currently no pure psychometric tests of spatial orientation ability.

One goal of this study was to develop valid tests of spatial orientation ability. On the basis of experimental studies of perspective taking (e.g., Hintzman, O'Dell, \& Arndt, 1981; Rieser, 1989; Shelton \& McNamara, 1997), we developed tests in which a person is shown a two- dimensional array of objects, imagines taking a perspective within the array, and indicates the direction to a target object from that perspective. We analyzed patterns of errors on these tests and verbal protocols to examine whether the dominant strategy used in these tests involves imagining reorienting oneself within the display, rather than mentally rotating the display.

A second goal of the study was to examine the extent to which object manipulation abilities and spatial orientation ability are separable. We analyzed performance on tests of object manipulation abilities and spatial orientation abilities, using confirmatory factor analysis (CFA). This method allowed us to test hypothesized factor structures and compare alternative models statistically (Kline, 1998). If object manipulation and spatial orientation abilities tap the same underlying construct, a model with a single spatial factor should provide a sufficiently good fit to the data. If, however, object manipulation and spatial orientation abilities are distinct, a model with a single spatial factor will significantly deviate from the data. In contrast, a two-factor model that assumes their separability should provide a good fit to the data. The correlation between these two factors then provides an estimation of the degree to which the two abilities are related.

\section{EXPERIMENT 1}

\section{Method}

Participants. The participants were 71 undergraduate students recruited from the psychology subject pool at the University of California, Santa Barbara.

Materials. The materials consisted of seven paper-and-pencil tests of spatial abilities. Object manipulation abilities were assessed by using the Card Rotation Test, the Cube Comparison Test, ${ }^{2}$ and the Paper Folding Test (Ekstrom, French, \& Harman, 1976). The Card Rotation Test requires participants to view a two-dimensional target figure and judge which of the five alternative test figures are planar rotations of the target figure (as opposed to its mirror image) as quickly and as accurately as possible. In the Cube Comparison Test, each item presents two drawings of cubes, with letters and numbers printed on their sides. Participants must judge whether the two drawings could show the same cube. Items in the Paper Folding Test show drawings of two or three folds made in a square sheet of paper. The final drawing shows a hole being punched in the folded paper. The task is to select one of five drawings that shows how the punched sheet would appear when fully opened.

The participants were presented with the Guilford-Zimmerman Spatial Orientation Test as well (Guilford \& Zimmerman, 1948). The test consists of items presenting two pictures of a lake and scenery as seen from looking out over the prow of a motorboat, which has moved slightly between pictures. The task is to select one of five diagrams that represents how the boat has moved. Each diagram shows a dot representing the old position of the prow and a dash representing the new position. Changes include any combination of heading change (i.e., rotation) and of forward translation and sideward translation of the boat.

To measure students' spatial orientation ability, we developed two perspective-taking tests. In the first test (the Object Perspective Test), a configuration of seven objects was drawn on a $8.5 \times 11$ in. sheet of paper (see Figure 1A). This display was fixed to the wall directly in front of each participant and was visible at all times. Each participant also received an answer booklet with a separate page for each of the test items. On each item, the participant was 
asked to imagine being at the position of one object in the display (the station point) facing another object (defining the imagined heading or perspective within the array) and was asked to indicate the direction to a third (target) object. The page of the answer sheet for each test item showed a picture of a circle, in which the imagined station point was drawn in the center of the circle and the imagined heading was drawn as an arrow pointing vertically up. The task was to draw another arrow from the center of the circle indicating the direction to the target object (e.g., "imagine you are standing near the stop sign facing the house; point to the traffic light"). The participants were prevented from physically rotating either the display showing the object configuration or their answer sheets.

In the second perspective-taking test (the Map Perspective Test), a map showing five landmarks was drawn on a $8.5 \times 11$ in. sheet of paper (see Figure 1B) and was pinned to the wall in front of the participant. The method of responding, by drawing an arrow on a circle, was the same as that for the Object Perspective Test.

The direction of the target object relative to the heading was varied systematically by dividing the circle into eight sections $\left(0^{\circ}-45^{\circ}\right.$, $45^{\circ}-90^{\circ}$, and so on). Both perspective-taking tests consisted of 10 test items, with the correct answer for at least 1 item being within each of the eight sections. The score for each item was the absolute deviation in degrees between the participant's response and the correct direction to the target (absolute directional error). A participant's total score was the average deviation across all items. If a participant did not point to any target direction, a score of $90^{\circ}$ was assigned for that item (i.e., chance performance, since the absolute angular deviation can range from $0^{\circ}$ to $180^{\circ}$ ). This occurred on $5 \%$ of all observations.

In addition, the participants were administered the Santa Barbara Sense of Direction Scale (SBSOD; Hegarty, Richardson, Montello, Lovelace, \& Subbiah, 2001). This self-report scale consists of 15 statements designed to measure a person's judgment of his or her own spatial orientation ability. Examples of typical statements are "my sense of direction is very good" and "I very easily get lost in a new city." Participants respond by circling a number from 1 (strongly disagree) to 7 (strongly agree) to indicate their level of agreement with each statement. All the items were scored so that a higher rating indicated a better self-report sense of direction (i.e., the scoring on negatively stated items was reversed).

Procedure. The participants were tested in groups of up to 6 students per session. They completed the Object Perspective Test, the Guilford-Zimmerman Spatial Orientation Test, the Cube Comparison Test, the Paper Folding Test, the Card Rotation Test, the Map Perspective Test, and the SBSOD, in that order. Each of the tests was administered according to its standard instructions, including time limits. The participants were allowed 4 min to complete each perspective-taking test. Completion of the SBSOD was not timed.

\section{Results and Discussion}

Descriptive statistics and measures of internal reliability are given in Table 1.

First, we examined whether the patterns of the participants' responses on our paper-and-pencil perspective tests were comparable with patterns of responses found for experimental perspective-taking tasks involving real arrays of objects.

Note that the perspective-taking tasks involve mental transformations over two angles: (1) the angle between the orientation of the array and the perspective to be imagined and (2) the angle between the imagined perspective and the direction to the target object. Both of these transformations contribute to task difficulty. Errors and response times increase with the angular deviation of the imagined perspective from the orientation of the array (e.g., Hintzman et al., 1981; Rieser, 1989; Shelton \& McNamara, 1997), and when imagining taking a particular perspective, people are faster and more accurate in naming and pointing to objects in front and behind them, as compared with items to the right or left (e.g., D. Bryant \& Tversky, 1999; Hintzman et al., 1981). Therefore, we examined how each of these angles contributed to performance on our paper-and-pencil perspective-taking tests.

Pointing accuracy as a function of imagined heading. Consistent with experimental research (Hintzman et al., 1981; Rieser, 1989; Shelton \& McNamara, 1997), absolute angular error increased with the angular deviation of one's imagined heading (perspective) from the orientation of the array (see Figure 2). The data plotted in Figure 2 present mean absolute pointing error as a function of this angular deviation, combining items from the two tests. The linear regression in Figure 2 was reliable $[F(1,19)=8.12, p<.01]$.

Pointing accuracy as a function of pointing direction. We categorized all items from both perspectivetaking tests as front, right-front, right, and so forth according to the direction to the target from the participant's imagined heading (eight sections of $45^{\circ}$ ). Figure 3 presents absolute pointing error for targets in these eight sections. An analysis of variance showed a significant effect of pointing direction $[F(7,490)=12.93, p<.001]$. Planned contrasts revealed that pointing accuracies for front and back responses were significantly higher than those for all the others $(p<.001)$. Front responses were also more accurate than back responses $(p<.01)$, and right-front and left-front responses were significantly more accurate than right-back and left-back responses, respectively $(p<.01)$. These results are very similar to the response profiles observed by Hintzman et al. (1981) for a similar pointing task and by D. Bryant and Tversky (1999) for time to identify objects from an imagined perspective.

In summary, the results above provide evidence that error patterns on paper-and-pencil perspective-taking tests are similar to those on experimental perspectivetaking tasks. However, this is not sufficient to establish that the perspective-taking tests are solved by a strategy that involves mentally reorienting oneself, rather than mentally rotating the stimuli. The increase in pointing error as a function of imagined heading is consistent with both a perspective-taking strategy and a mental rotation strategy. Although the pattern of accuracy as a function of pointing direction is suggestive of a body-centered coordinate system, it is possible that front and back responses are easier because they can be solved by a simpler strategy (i.e., noting that the three objects mentioned in an item form a straight line).

Imagined perspective taking usually leads to systematic errors that reflect the symmetry of the coordinate system of the body, whereas errors in rotation problems are not systematic (Huttenlocher \& Presson, 1973, 1979; Presson, 1982). In order to establish the validity of our paperand-pencil perspective-taking tests, we quantified errors 
A

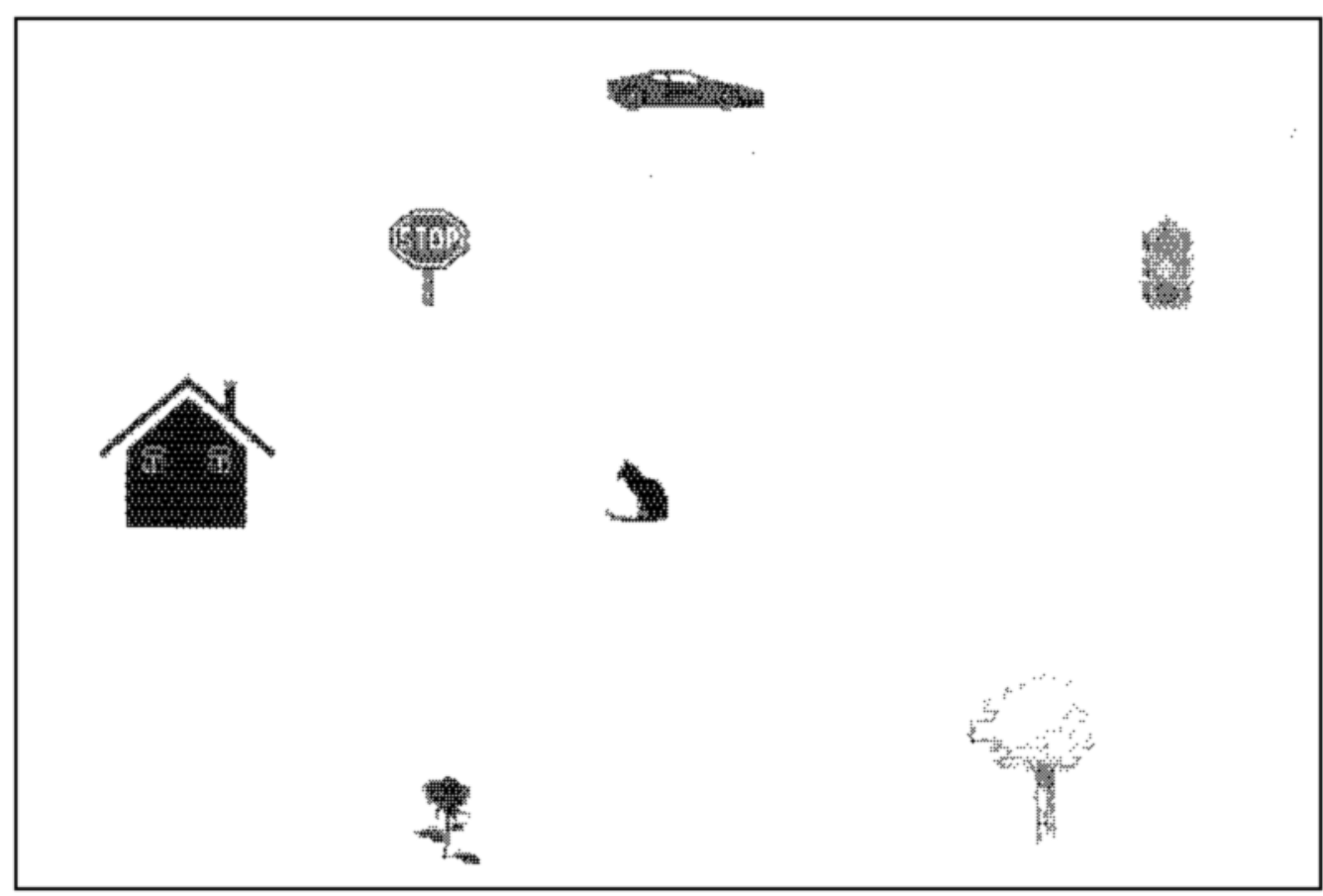

B

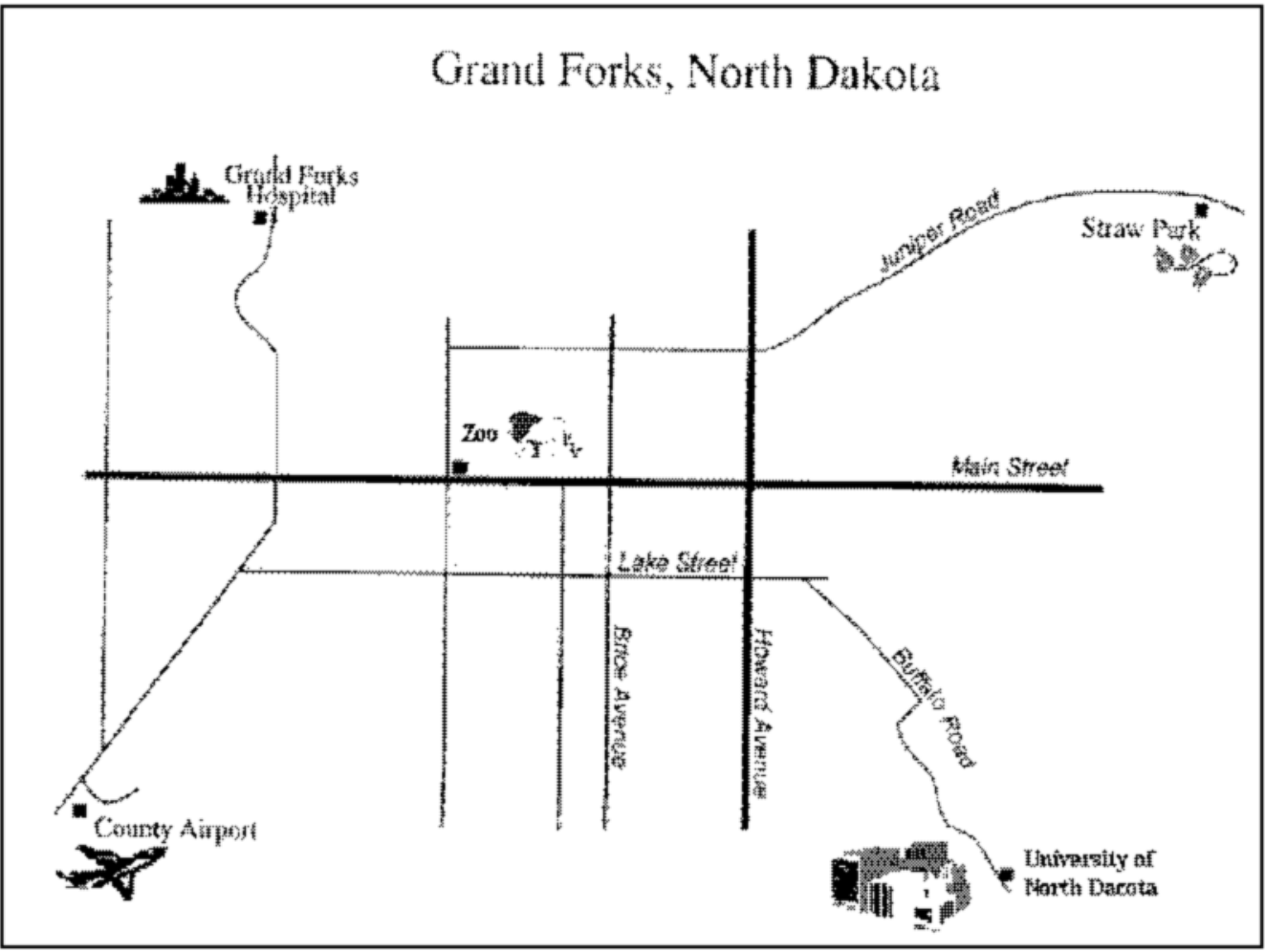

Figure 1. Configuration of objects presented to subjects in (A) the Object Perspective-Taking Test and (B) the Map Perspective-Taking Test. 
Table 1

Descriptive Statistics and Measures of Internal Reliability for the Spatial Abilities Measures

\begin{tabular}{lrrcc}
\hline \multicolumn{1}{c}{ Test } & Mean & \multicolumn{1}{c}{$S D$} & Observed Range & Reliability \\
\hline Card Rotation & 110.9 & 33.8 & $0-156$ & .80 \\
Cube Comparison & 18.6 & 11.5 & $0-40$ & .84 \\
Paper Folding & 11.6 & 3.9 & $1.5-20$ & .84 \\
Guilford-Zimmerman & 17.1 & 10.9 & $0-50$ & .88 \\
Object Perspective & 33.2 & 23.3 & $5-95$ & .83 \\
Map Perspective & 34.4 & 25.3 & $5-120$ & .85 \\
Sense of Direction & 3.5 & 1.6 & $1-6$ & .88 \\
\hline
\end{tabular}

that reflect the symmetry of the coordinate system of the physical body (left/right and front/back axes of the observer's body). We refer to these as reflection errors.

Reflection errors. We drew a circular plot of participants' responses for each perspective-taking item. For instance, Figure 4A shows the distribution of responses on Item 1 from the Object Perspective Test ("imagine you are standing at the house and facing the stop sign; point to the traffic light"). Each triangle represents the end of the arrow drawn by 1 participant. This item requires the participant to imagine changing his/her orientation by $50^{\circ}$, and the correct answer is in the upper right quadrant (quadrant I). As Figure 4A shows, all the participants' responses fall into the correct quadrant of the circle, and the responses are approximately normally distributed around the modal response.

Now consider Figure 4B, representing participants' responses on Item 3 of the Object Perspective Test. This item asks the participant to imagine standing at the house, facing the flower, and to point to the traffic light $\left(148^{\circ}\right.$ difference between the imagined perspective and the orientation of the array). The correct answer to this item falls in the left upper quadrant (quadrant II). Although most responses are in this quadrant, some responses also fall into other quadrants. Furthermore, the incorrect responses are not random but are grouped either in the right upper quadrant (quadrant I), suggesting a reflection through the vertical axis, or in the lower left quadrant (quadrant III), suggesting a reflection through the horizontal axis. Quadrant I errors would be produced if the participants encoded the objects in a body-centered frame of reference and confused left and right, whereas quadrant III errors would be produced if they confused front and back. Quadrant IV errors would result if the participants confused between both left and right and front and back.

To examine whether these errors are characteristic of other trials, we first classified a response as an error if it fell in the wrong quadrant. We then classified errors on each trial as reflection errors if they were within $20^{\circ}$ of a response that was a reflection of the correct response through the horizontal, vertical, or both axes. Figure 5 shows the mean number of participants who made reflection errors on items for different imagined headings and the mean number of participants who made other errors (errors falling in the incorrect quadrant, but not within $20^{\circ}$ of a reflection of the correct answer). The number of reflection errors increases significantly with the angular deviation of imagined heading (perspective) from the orientation of the array, whereas the number of other errors does not. Items involving perspective changes of less than $90^{\circ}$ resulted in significantly fewer reflection errors (mean number of quadrant errors is $2.5, S D=2.5$ ), as compared with the items involving orientation changes of more than $90^{\circ}$ [mean number of quadrant errors is $10.71, S D=$ $5.7 ; F(1,19)=11.27, p<.01]$. In contrast, the number of

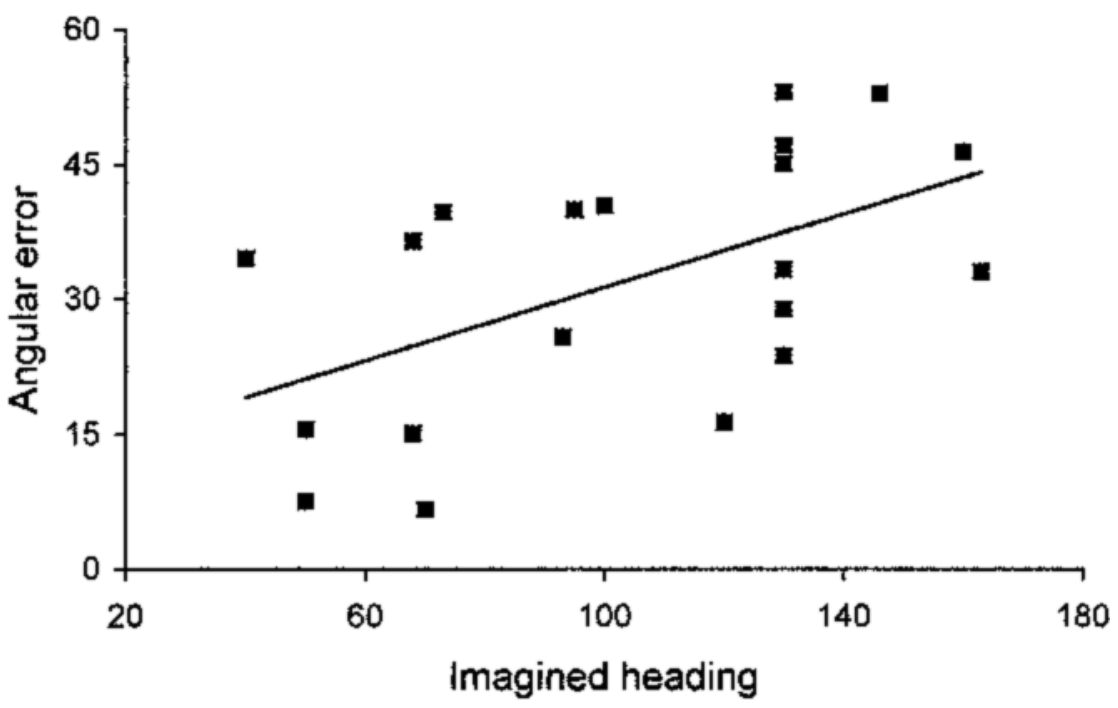

Figure 2. Absolute pointing error as a function of the angular deviation of imagined heading (perspective) from the orientation of the array. (The solid curve represents the regression line.) 


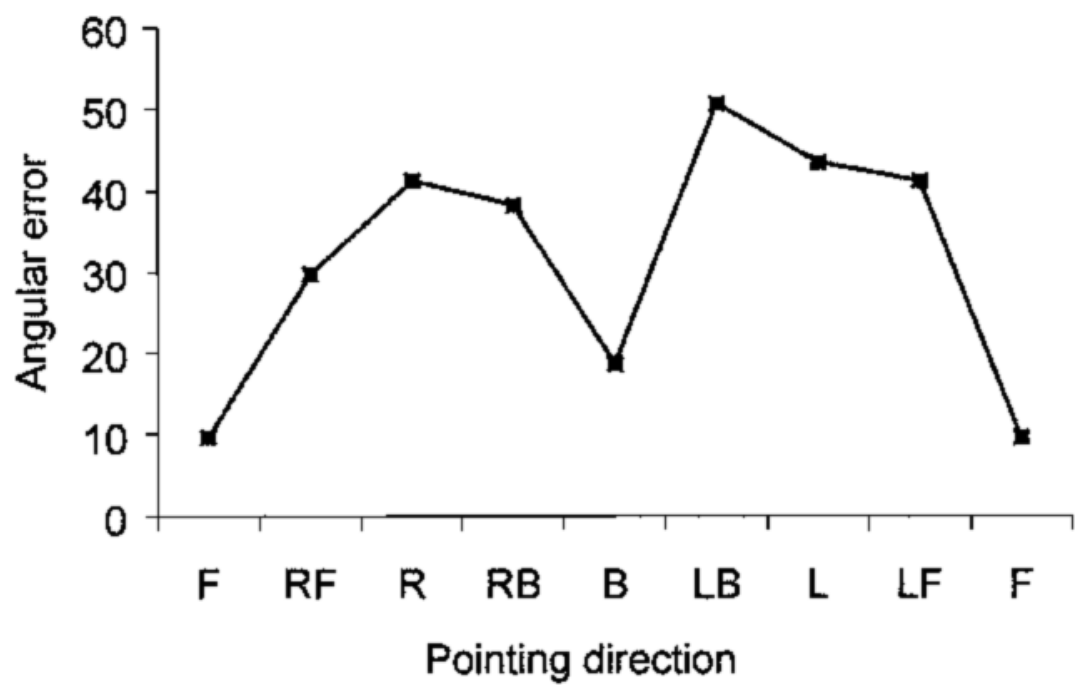

Figure 3. Absolute pointing error as a function of the direction to the target from one's imagined heading. F, front; $R F$, right front; $R$, right; $R B$, right back; $B$, back; $L B$, left back; L, left; and LF, left front.

other errors did not differ significantly with orientation change $[F(1,19)=0.46]$.

The results above suggest that people use the egocentric frame of reference to solve perspective-taking tasks. Difficulties in specifying right-left and back-front directions to the target would not arise if the participants performed the task primarily with an object manipulation strategy. Furthermore, if the participants gave up and guessed on difficult trials, this would lead to an increase in all types of errors, and not just reflection errors.

There were few reflection errors for items involving a perspective change of less than $90^{\circ}$. It is possible that these items were performed with a perspective-taking strategy but were significantly easier than items involving a larger change of perspective. Alternatively, the participants might have used other strategies to solve items involving a perspective change of less than $90^{\circ}$. To discriminate between these alternatives, in Experiment 2, we asked participants to think aloud while they solved perspective-taking items and classified the strategies that they reported.

\section{EXPERIMENT 2}

\section{Method}

Participants. The participants were 8 undergraduate students who had not participated in Experiment 1. They were recruited from the psychology subject pool at the University of California, Santa Barbara.

Materials. Two displays, identical to those used in the Object Perspective and Map Perspective Tests, were fixed on the wall directly in front of the participant. The participant received an answer booklet with a page for each of 13 test items. The format of the items and the method of responding were the same as those in Experiment 1 . Nine items referred to the object display and 4 items referred to the map display. Six items required the participant to change his/her perspective less than $90^{\circ}, 2$ required a change of $90^{\circ}$, and 5 other items required a perspective change of more than $90^{\circ}$.

Procedure. The participants were first told to think aloud while solving each item, and after answering, they were also asked specifically whether they imagined changing their perspective or used another strategy. We videotaped the participants in order to record verbalizations, hand movements, and drawings.

\section{Results}

We identified three strategies used by the participants to perform the perspective-taking tasks.

1. Finding the angle. Those participants who used this strategy reported that they just saw the angle or tilted their heads to see the angle but did not indicate any imagined perspective change or rotation of the array. The following transcripts are examples of responses that were classified as indicative of this strategy: "I could see the angle without imagining myself there. ..," "I just tilted my head . . . I saw the line and the angle. I didn't really imagine myself there ...." "I just see that the house, stop sign, and car form a straight line, so I just think of it as that ... and in this way put them on the circle...."

2. Mental rotation. The participants were classified as using this strategy if they reported that they mentally rotated the display or angle connecting three objects (station point, heading, and target). The following examples illustrate typical descriptions of the above strategy: "If I did imagine something, it was that the paper turned like this and the line was here ...," "I imagined the paper turning ... actually the angle between three objects turning ... it's easier than imagining turning myself. ..."

3. Perspective taking. The participants scored as using this strategy explicitly reported that they imagined reorienting themselves while solving the task. Typical responses that were scored as perspective taking were as 
A. Imagine you are at the house and facing the stop sign, point to the traffic light

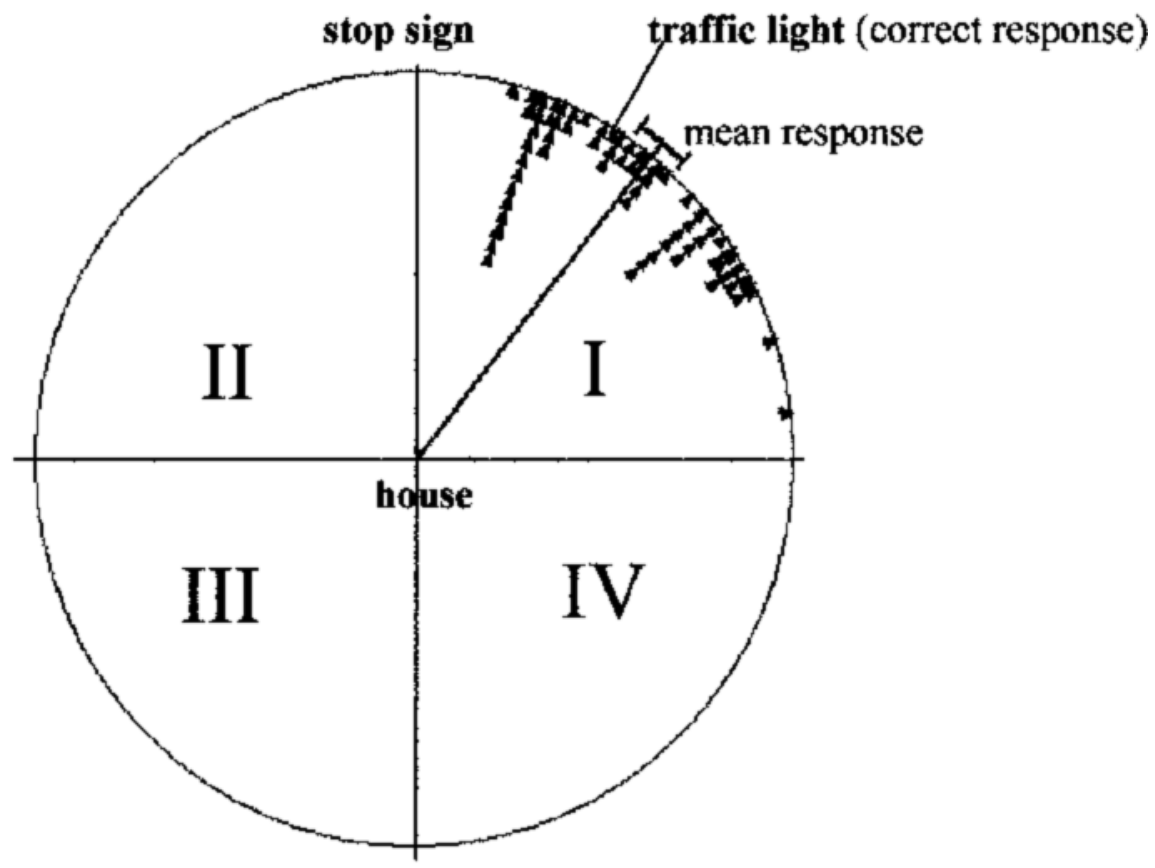

B. Imagine you are at the house and facing the flower, point to the traffic light

\section{flower}

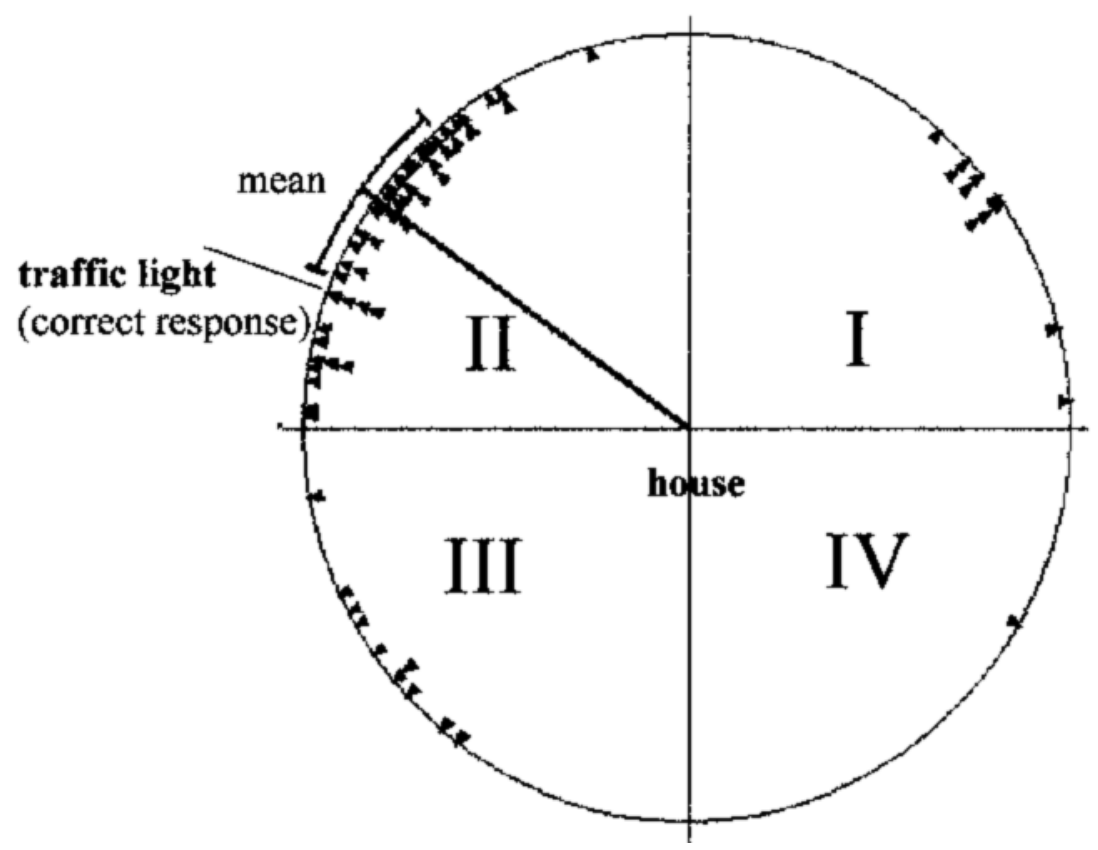

Figure 4. Distribution of participants' responses on (A) Item 1 of the Object Perspective Test and (B) Item 3 of the Object Perspective Test. Triangles represent the ends of the arrows showing the direction to the target object drawn by each subject. 


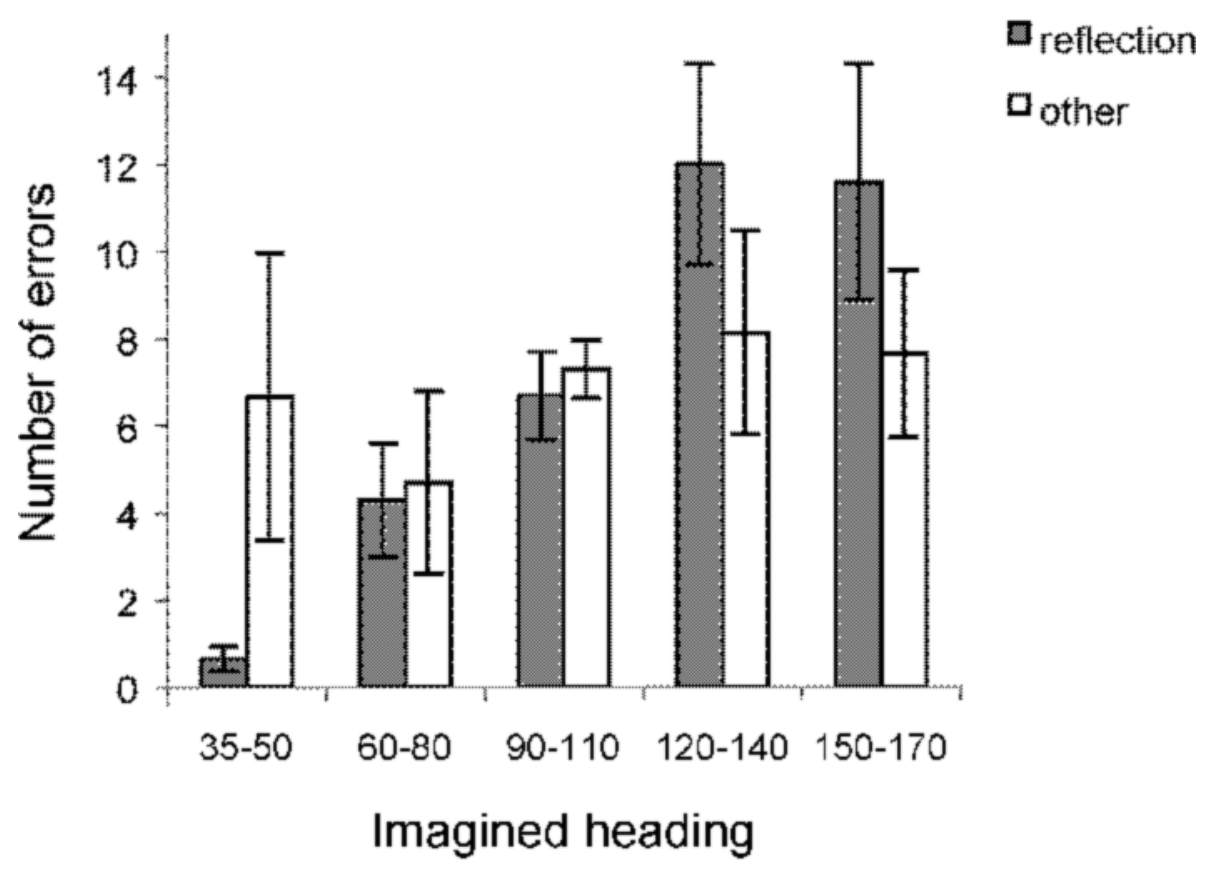

Figure 5. The mean number of reflection errors and other errors as a function of the deviation of imagined heading from the orientation of the display. Error bars represent the standard error of the mean.

follows: "I' $m$ trying to spin myself in my head ...," "I'm facing the car, and I' $m$ trying to see where it would be in relation to my right shoulder. I'm trying to picture myself there ...," "I had to turn myself around because it's facing the opposite direction. ..."

Two independent raters, who were unaware of the purpose of the study, viewed the videotapes and classified participants' responses on each item as using one of the three strategies. The agreement between raters regarding the type of strategy used was .89. Disagreements were settled by consensus of the two raters.

The first two strategies, finding the angle and mental rotation, might be classified as object manipulation strategies. Those who reported the angle strategy seemed to retrieve the interobject relations from the initial orientation of the array and then impose them on the orientation of the answer circle. Those who used the mental rotation strategy explicitly described computing the angle between the station point, heading, and target and then rotating the angle.

In contrast, participants reporting the perspectivetaking strategy mentioned changes of their own orientation. Their protocols indicated that they encoded the objects in the array or map with respect to their own bodies (self-object representational system), as the following examples illustrate: "I'm standing here, my shoulders face here, and I see the stop light in relation to my shoulders. Once I position my shoulders, I can judge the angles and right from left ...., "I had to think more because it was on different sides. I had to think: which is my arm pointing to?" Reports of left-right or back-front discrimination accompanied the majority of trials in which a participant reported a perspective-taking strategy (52 out of 64 reports). When other strategies were used, no left-right or back-front discriminations were reported.

As is shown in Table 2, most participants reported the angle strategy for items requiring reorientation of less than $90^{\circ}$. However, for tasks requiring a perspective change of more than $90^{\circ}$, the dominant strategy was perspective

Table 2

Number of Participants Who Were Classified as Using the Different Strategies for Individual Perspective-Taking Items in Experiment 2

\begin{tabular}{cclccc}
\hline & Perspective & Pointing & \multicolumn{3}{c}{ Strategy } \\
\cline { 4 - 6 } Item & Change $\left({ }^{\circ}\right)$ & Direction & Perspective & Angle & Rotation \\
\hline 1 & 45 & right-front & 3 & 5 & 0 \\
2 & 45 & front & 2 & 6 & 0 \\
3 & 45 & right-front & 2 & 6 & 0 \\
4 & 50 & back & 3 & 5 & 0 \\
5 & 50 & right-back & 4 & 4 & 0 \\
6 & 70 & left-back & 3 & 5 & 0 \\
7 & 90 & right-front & 6 & 1 & 1 \\
8 & 90 & left-front & 6 & 1 & 1 \\
9 & 130 & left & 7 & 0 & 1 \\
10 & 130 & back & 7 & 0 & 1 \\
11 & 145 & left-front & 7 & 0 & 1 \\
12 & 145 & left-back & 7 & 0 & 1 \\
13 & 150 & right-front & 7 & 0 & 1 \\
\hline
\end{tabular}

Note-Items are classified by the change of perspective required by the item and the direction of pointing from the imagined heading. 
Table 3

Correlations Between the Spatial Ability Measures

(Pearson Correlation Coefficients, $N=71$ )

\begin{tabular}{lccccccc}
\hline & Object & Map & Cube & Card & Paper & & \\
Task & Perspective & Perspective & Comparison & Rotation & Folding & Guilford-Zimmerman & SBSOD \\
\hline Object & - & $.72 \dagger$ & $.50 \dagger$ & $.27 \dagger$ & $.47 \dagger$ & $.34 \dagger$ & $.28 \dagger$ \\
Map & & - & $-.49 \dagger$ & $.18^{*}$ & $.45 \dagger$ & $.32 \dagger$ & $.19^{*}$ \\
Cube & & & - & $.52 \dagger$ & $.57 \dagger$ & $.38 \dagger$ & $.29 \dagger$ \\
Card & & & & - & $.46 \dagger$ & $.28 \dagger$ & .09 \\
Paper & & & & & - & $.35 \dagger$ & .12 \\
G-Z & & & & & & - & $.25^{*}$ \\
\hline
\end{tabular}

Note-Scores on the Object and Map Tests reported in the table are for a restricted set of items (more than $90^{\circ}$ ) and are linearly transformed so that higher values correspond to better performance. SBSOD, Santa Barbara Sense of Direction Scale. ${ }^{*} p<.05 . \quad \dagger p<.01$.

taking. (Only 1 of the 8 participants changed from the angle strategy to mental rotation.) A paired-sample $t$ test revealed that the proportion of perspective-taking strategies reported by the participant on items with an orientation change of more than $90^{\circ}(M=.88, S D=.24)$ was significantly higher than the proportion of perspectivetaking strategies reported by the participants to items with an orientation change of less than $90^{\circ}[M=.35$, $S D=.18 ; t(7)=9.91, p<.001)$.

\section{Conclusions}

The results of the protocol study support the validity of the perspective-taking tests as tests of spatial orientation, by showing that the dominant strategy used on these items is to imagine oneself reoriented within the array. However, this is true only for items that require a perspective change of more than $90^{\circ}$. In contrast, most items that require a perspective change of less than $90^{\circ}$, are solved by object manipulation strategies.

\section{CONFIRMATORY FACTOR ANALYSIS}

Having established the validity of the perspectivetaking tests, we now turn to the second goal of the study, which is to examine whether spatial orientation ability, as measured by our perspective-taking tests, is separable from object manipulation abilities (i.e., spatial visualization and spatial relations). To examine this question, we performed a CFA on the data from Experiment 1. On the basis of the results of the protocol analysis, we excluded items requiring a perspective change of less than $90^{\circ}$ from this analysis (five items in the Object Perspective Test and three items in the Map Perspective Test). Scores on the restricted set of items were linearly transformed so that higher values corresponded to better performance (by subtracting the average error score for each participant from $180^{\circ}$ ). The internal reliability of the resulting Object Perspective Test was .82, $(M=137.26$, $S D=32.77$ ) and of the resulting Map Perspective Test was $.82(M=145.22, S D=28.27)$.

Table 3 shows the correlations between the different spatial ability tests. The measures are significantly correlated with each other (with the exception of the SBSOD, which does not correlate with Card Rotation or Paper Folding).
According to our model, there are two separable spatial factors: object manipulation ability and spatial orientation ability. We assumed that Card Rotation, Cube Comparison, and Paper Folding would load on the object manipulation spatial factor and that Object Perspective and Map Perspective would load on the spatial orientation factor. The SBSOD was also assumed to load on the spatial orientation factor, because similar self-report measures have been shown to correlate with an ability to take a perspective in a familiar environment and point to a landmark in that environment (K. Bryant, 1982; Sholl, 1988).

There has been doubt about whether the GuilfordZimmerman Spatial Orientation Test measures spatial orientation, rather than other spatial abilities (Barratt, 1953; Carpenter \& Just, 1986; Carroll, 1993). To examine this question, we tested a preliminary two-factor model, in which this test was allowed to load on both factors, and found that it loaded significantly only on the object manipulation factor ( $15 \%$ overlapping variance). Thus, in our final version of the two-factor model, we considered this test as loading only on object manipulation.

The estimated two-factor model, complete with factor loadings, is illustrated in Figure 6. The numbers above the straight, single-headed arrows are standardized factor loadings, and the arrows at the end of the shorter, singleheaded arrows represent error terms. All these numbers can be interpreted as standardized regression coefficients.

Values of fit indices for the two-factor model are reported in Table 4. All indices suggest that the two-factor model fits the data. This model produced a nonsignificant $\chi^{2}(13)=13.16, p=.44$, indicating that the model did not significantly deviate from the data. In addition, the Bentler Comparative Fit Index (CFI) is .99, well above the commonly used criterion of .90 for a good fit; and the standardized root-mean square residual (SRMR) is .012, well below the criterion of .05 .

The two-factor model indicates that perspective taking and spatial visualization are highly correlated with each other, as would be expected. But are these factors identical? We considered an alternative single-factor model, in which the correlation between the two factors was fixed to 1 . The fit indices for this model are shown in Table 4 . The overall chi-square for the single-factor model was significant $\left[\chi^{2}(14)=35.68, p=.001\right]$, indicating that the 


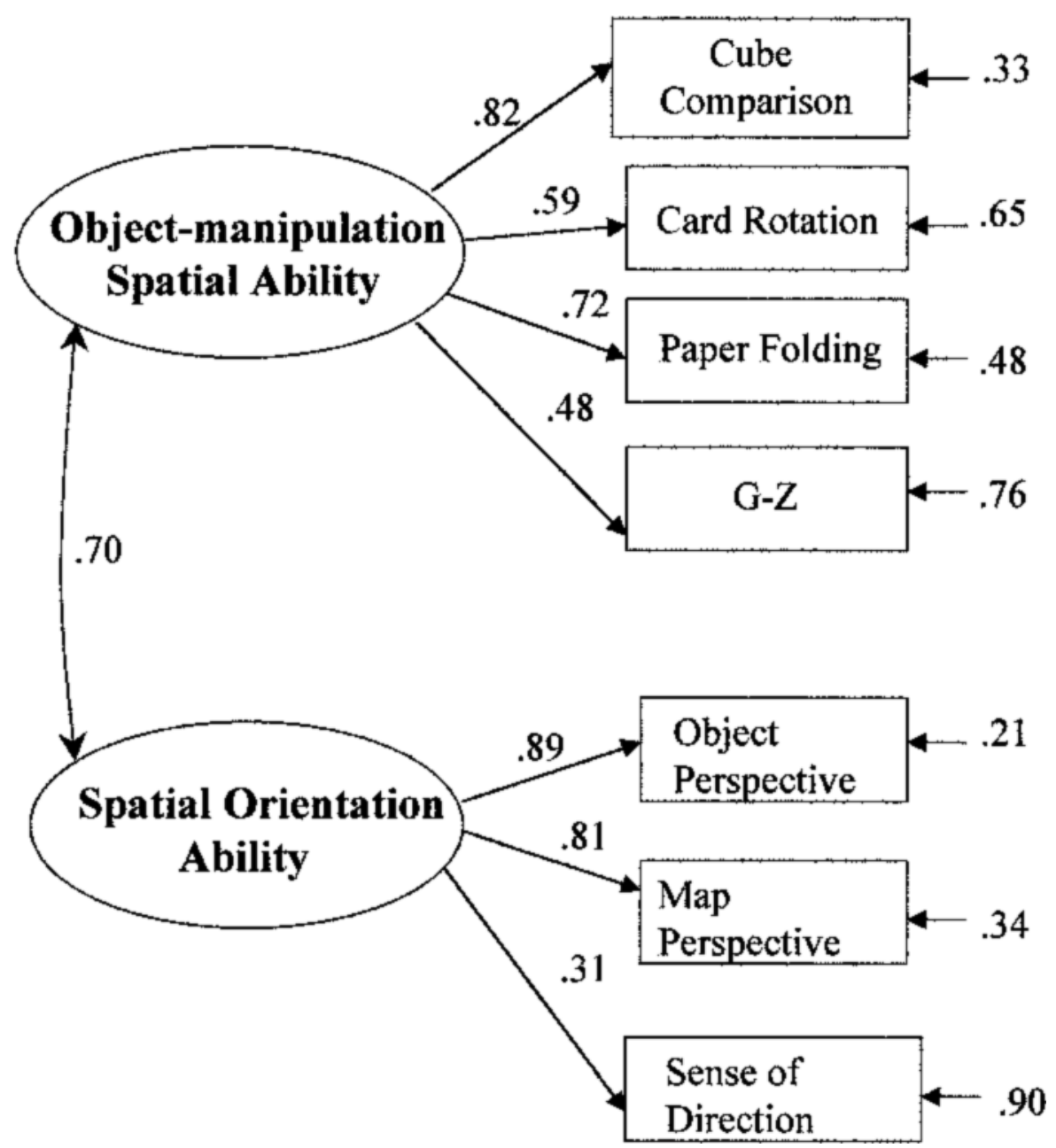

Figure 6. The results of the confirmatory factor analysis.

model significantly deviates from the data. In addition, the values of CFI (.86) and SRMR (.139) do not meet the criteria for a good fit. Thus, the single-factor model does not explain the data. Moreover, a chi-square difference test comparing the fit of the two models indicates that the overall fit of the two-factor model is significantly greater than that of the single-factor model $\left[\chi^{2}(1)=\right.$ $22.53, p<.01]$.

\section{GENERAL DISCUSSION}

We developed new spatial orientation tests, based on tasks used in experimental studies of perspective taking. The patterns of students' responses on our tests are very similar to response profiles found in previous experimental studies. Furthermore, we quantified errors characteristic of a perspective-taking strategy that involve confusion between left/right as well as back/front pointing directions. The number of these errors increased significantly when the angle between the orientation of the array and the perspective to be imagined exceeded $90^{\circ}$. These results suggest that people use a perspective-taking strategy to solve items that require changing imagined heading by more than $90^{\circ}$. These systematic errors would not be predicted if people performed the perspective-taking tasks mainly with an object manipulation strategy.

The results of the protocol analysis also support the claim that perspective taking is the prevalent strategy used to solve Object Perspective and Map Perspective items that involve a perspective change of more than $90^{\circ}$. Although it is possible to solve these items by mentally rotating the display, rather than by imagining oneself reoriented in space, 7 of the 8 participants interviewed in Experiment 2 reported a perspective-taking strategy.

The second goal of our study was to establish whether object manipulation spatial abilities (i.e., spatial visualization and spatial relations) can be dissociated from perspective-taking spatial abilities. The CFA indicated that a model assuming that these are separate abilities is a better fit to the data than a model that assumes a single 
Table 4

Comparison of the Single-Factor and Two-Factor Models

\begin{tabular}{lcccccccc}
\hline \multicolumn{1}{c}{ Model } & $\chi^{2}$ & $d f$ & $\chi^{2} / d f$ & $\chi^{2}$ difference & $d f_{\text {difference }}$ & CFI & NNFI & SRMR \\
\hline Single-factor & $35.68^{*}$ & 14 & 2.62 & - & - & .86 & .80 & .14 \\
Two-factor & 13.16 & 13 & 0.16 & $22.52 *$ & 1 & .99 & .93 & .01 \\
\hline
\end{tabular}

Note-CFI, Bentler Comparative Fit Index; NNFI, Bentler-Bonett Non-Normed Fit Index; SRMR, standardized root-mean squared residual. $* p<.01$.

spatial factor. Although they are correlated, object manipulation and spatial orientation abilities can be separated.

In contrast, the Guilford-Zimmerman Spatial Orientation Test, which is the most commonly used test of spatial orientation ability, could not be distinguished from object manipulation spatial abilities. We suggest that the Guilford-Zimmerman Test is a less valid test of spatial orientation because it does not involve large changes in perspective (only differences of about $30^{\circ}$ ). Our data suggested that people do not use a perspective-taking strategy for items less than $90^{\circ}$. Consistent with this interpretation, Barratt (1953) found that many people report solving items in the Guilford-Zimmerman Test by an object manipulation strategy. It seems that in order to measure individual differences in spatial orientation abilities, it is necessary to test people on items that involve orientation changes of $90^{\circ}$ or more.

The fact that SBSOD loads on the perspective-taking factor is very preliminary evidence that performance in our pencil-and-paper perspective-taking tests might be related to perspective taking in large-scale space. This evidence is relatively weak, because the loading is not high and SBSOD is not a direct measure of performance. Further research is required to examine the relation of perspective-taking tests to other spatial tasks, such as taking a perspective in an array of real objects and navigation in large-scale space.

In conclusion, this research indicates that a person's ability to mentally manipulate a visual stimulus from a stationary point of view (object manipulation ability) does not reflect his/her ability to reorient him/herself in space (spatial orientation ability). Although they are highly correlated, object manipulation and perspective-taking tests do not appear to reflect the same construct. Failure to find a distinction between these abilities in the psychometric literature to date might be attributed to the fact that previous spatial orientation tests used were not pure measures of this ability.

\section{REFERENCES}

BARRATt, E. S. (1953). An analysis of verbal reports of solving spatial problems as an aid in defining spatial factors. Journal of Psychology, 36, 17-25.

Borich, G. D., \& Bauman, P. M. (1972). Convergent and discriminant validation of the French and Guilford-Zimmerman spatial orientation and spatial visualization factors. Educational \& Psychological Measurement, 32, 1029-1033.

Bryant, D., \& TVERsKy, B. (1999). Mental representations of perspective and spatial relations from diagrams and models. Journal of Experimental Psychology: Learning, Memory, \& Cognition, 25, 137-156. BRYANT, K. (1982). Personality correlates of sense of direction and ge- ographical orientation. Journal of Personality \& Social Psychology, 43, 1318-1324.

CArpenter, P. A., \& Just, M. A. (1986). Spatial ability: An informationprocessing approach to psychometrics. In R. J. Stenberg (Ed.), Advances in the psychology of human intelligence (Vol. 3, pp. 221-252). Hillsdale, NJ: Erlbaum.

CARroll, J. (1993). Human cognitive abilities: A survey of factoranalytical studies. New York: Cambridge University Press.

EAston, R. D., \& Sholl, M. J. (1995). Object-array structure, frames of reference, and retrieval of spatial knowledge. Journal of Experimental Psychology: Learning, Memory, \& Cognition, 21, 483-500.

Ekstrom, R. B., French, J. W., \& Harman, H. H. (1976). Manual for Kit of Factor Referenced Cognitive Tests. Princeton, NJ: Educational Testing Service.

Eliot, J., \& S Mith, I. M. (1983). An international directory of spatial tests. Windsor Berkshire, U.K.: NFER-Nelson.

Guilford, J. P., \& Zimmerman, W. S. (1948). The Guilford-Zimmerman Aptitude Survey. Journal of Applied Psychology, 32, 24-34.

Hegarty, M., Richardson, A. E., Montello, D. R., Lovelace, K., \& SubBiah, I. (2001). The Santa Barbara Sense of Direction Scale. Unpublished manuscript, University of California, Santa Barbara.

Hintzman, D. L., O’Dell, C. S., \& ARndt, D. R. (1981). Orientation in cognitive maps. Cognitive Psychology, 13, 149-206.

Huttenlocher, J., \& Presson, C. C. (1973). Mental rotation and the perspective problem. Cognitive Psychology, 4, 277-299.

Huttenlocher, J., \& Presson, C. C. (1979). The coding and transformation of spatial information. Cognitive Psychology, 11, 375-394.

KLINe, R. B. (1998). Principles and practice of structural equation modeling. New York: Guilford.

LoHman, D. F. (1988). Spatial abilities as traits, processes, and knowledge. In R. J. Sternberg (Ed.), Advances in the psychology of human intelligence (pp. 181-248). Hillsdale, NJ: Erlbaum.

McGee, M. G. (1979). Human spatial abilities: Psychometric studies and environmental, genetic, hormonal, and neurological influences. Psychological Bulletin, 86, 889-918.

Muller, R. U., Kubie, J. L., Bostock, E. M., Taube, J. S., \& Quirk, G. J. (1991). Spatial firing correlates of neurons in the hippocampus formation of freely moving rats. In J. Paillard (Ed.), Brain and space (pp. 269-333). Oxford: Oxford University Press.

O'KeEfe, J., \& NADEL, L. (1978). The hippocampus as a cognitive map. Oxford: Oxford University Press.

Paillard, J. (1991). Brain and space. Oxford: Oxford University Press. Presson, C. C. (1982). Strategies in spatial reasoning. Journal of Experimental Psychology: Learning, Memory, \& Cognition, 8, 243-251.

Price, L., \& Eliot, J. (1975). Convergent and discriminant validities of two sets of measures of spatial orientation and visualization. Educational \& Psychological Measurement, 35, 975-977.

RIESER, J. J. (1989). Access to knowledge of spatial structure at novel points of observation. Journal of Experimental Psychology: Learning, Memory, \& Cognition, 15, 1157-1165.

Shelton, A. L., \& McNamara, T. P. (1997). Multiple views of spatial memory. Psychonomic Bulletin \& Review, 4, 102-106.

SHoll, M. J. (1988). The relation between sense of direction and mental geographic updating. Intelligence, 12, 299-314.

Simons, D. J., \& WANG, R. F. (1998). Perceiving real-world viewpoint changes. Psychological Science, 9, 315-320.

Wraga, M., Creem, S. H., \& Profitt, D. R. (2000). Updating displays after imagined object and viewer rotations. Journal of Experimental Psychology: Learning, Memory, \& Cognition, 26, 151-168.

Zacks, J., Rypma, B., Gabrieli, J. D. E., Tversky, B., \& Glover, G. H 
(1999). Imagined transformations of bodies: An fMRI investigation. Neuropsychologia, 37, 1029-1040.

\section{NOTES}

1. Although there is strong evidence for a dissociation between tests of spatial visualization and speeded rotation in the psychometric literature (Carroll, 1993), several tests of each type must be included in a factor analysis in order for these abilities to emerge as separate factors.
In this study, we were more concerned with the dissociation between object manipulation and spatial orientation abilities, so we did not include enough tests for spatial visualization and speeded rotation to emerge as separate factors. Therefore, we refer to these tests collectively as tests of object manipulation ability.

2. The Card Rotation and Cube Comparison Tests, classified as speeded rotation tests by most researchers (see Carpenter \& Just, 1986; Carroll, 1993; Eliot \& Smith, 1983; Lohman, 1988), were called spatial orientation tests by Ekstrom, French, and Harmon (1976).

\section{APPENDIX}

Instructions and a Sample Item From the Paper Folding Test (Ekstrom et al., 1976)

In this test you are to imagine the folding and unfolding of pieces of papers. The figures on the left represent a square piece of paper being folded, and the last of these figures has one or two small circles drawn on it to show where the paper has been punched. One of the five figures on the right shows where the holes will be when the paper is unfolded. You are to decide which one of these figures is correct.

\section{Instructions and Sample Items From the Card Rotation Test (Ekstrom et al., 1976)}

Each problem in this test consists of one card on the left of a vertical line and eight cards on the right. You are to decide whether each of eight cards on the right is the same as or different from the card at the left. Mark the box beside the $\mathrm{S}$ if it is the same as the one at the beginning of the row. Mark the box beside the $\mathrm{D}$ if it is different from the one at the beginning of the row.

\section{Instructions and Sample Items From the Guilford-Zimmerman \\ Spatial Orientation Test (Guilford \& Zimmerman, 1948)}

In each item you are to note how the position of the prow of the boat has changed in the second picture from its original position in the first picture. To work each item: First, look at the top picture. See where the motor boat is headed. Second, look at the bottom picture and note the CHANGE in the boat's heading. Third, select and mark one of five diagrams to represent how the boat has moved. Each diagram shows a dot representing the old position of the prow and a dash representing the new position.

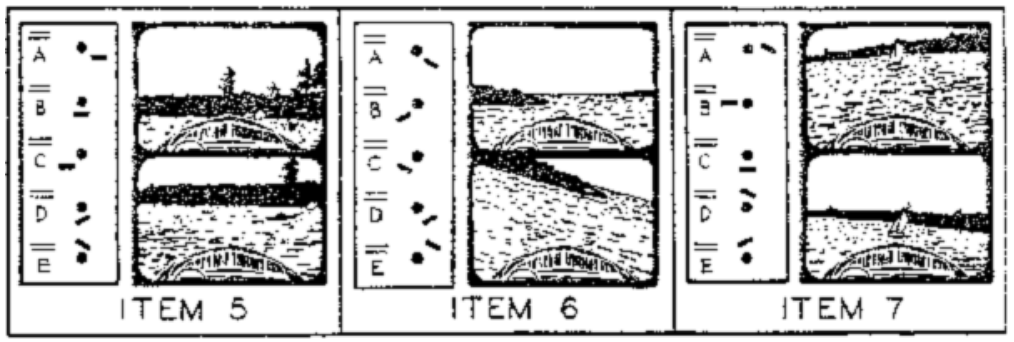

(Manuscript received August 4, 2000; revision accepted for publication February 2, 2001.) 